\title{
Estimating spatial mean root-zone soil moisture from point-scale observations
}

\author{
A. J. Teuling ${ }^{1}$, R. Uijlenhoet ${ }^{1}$, F. Hupet ${ }^{2}$, E. E. van $\operatorname{Loon}^{3}$, and P. A. Troch \\ ${ }^{1}$ Hydrology and Quantitative Water Management Group, Wageningen University, Wageningen, The Netherlands \\ ${ }^{2}$ Department of Environmental Sciences and Land Use Planning, Université Catholique de Louvain, Louvain-la-Neuve, \\ Belgium \\ ${ }^{3}$ Institute for Biodiversity and Ecosystem Dynamics, University of Amsterdam, Amsterdam, The Netherlands \\ ${ }^{4}$ Department of Hydrology and Water Resources, The University of Arizona, Tucson, Arizona, USA
}

Received: 3 April 2006 - Published in Hydrol. Earth Syst. Sci. Discuss.: 10 July 2006

Revised: 14 September 2006 - Accepted: 9 October 2006 - Published: 17 October 2006

\begin{abstract}
Root zone soil moisture is a key variable in many land surface hydrology models. Often, however, there is a mismatch in the spatial scales at which models simulate soil moisture and at which soil moisture is observed. This complicates model validation. The increased availability of detailed datasets on space-time variability of root-zone soil moisture allows for a posteriori analysis of the uncertainties in the relation between point-scale observations and the spatial mean. In this paper we analyze three comprehensive datasets from three different regions. We identify different strategies to select observation sites. For instance, sites can be located randomly or according to the rank stability concept. For each strategy, we present methods to quantify the uncertainty that is associated with this strategy. In general there is a large correspondence between the different datasets with respect to the relative uncertainties for the different strategies. For all datasets, the uncertainty can be strongly reduced if some information is available that relates soil moisture content at that site to the spatial mean. However this works best if the space-time dynamics of the soil moisture field are known. Selection of the site closest to the spatial mean on a single random date only leads to minor reduction of the uncertainty with respect to the spatial mean over seasonal timescales. Since soil moisture variability is the result of a complex interaction between soil, vegetation, and landscape characteristics, the soil moisture field will be correlated with some of these characteristics. Using available information, we show that the correlation with leaf area index or a wetness coefficient alone is insufficient to predict if a site is representative for the spatial mean soil moisture content.
\end{abstract}

Correspondence to: A. J. Teuling

(ryan.teuling@wur.nl)

\section{Introduction}

Soil moisture controls several processes at or near the land surface. The partitioning of rainfall into infiltration and runoff; the partitioning of available energy into latent and sensible heat; the drainage of water to groundwater and/or surface water; the growth of vegetation: all these processes are strong and nonlinear functions of soil moisture. Many forecasting applications rely on accurate soil moisture observations to predict these processes. However soil moisture observations are often available at the point-scale, while most models utilize effective parameters representative for the average soil and vegetation. Similar problems arise when point scale soil moisture observations are combined with flux measurements. Vertical fluxes of water and/or energy at several meters above the surface might be affected by spatial average soil moisture conditions at the scale of the flux footprint. At many stations, for instance in the FLUXNET network (Baldocchi et al., 2001), root zone soil moisture is monitored at a scale several orders of magnitude smaller than the corresponding flux footprint. When validating model simulations with point-scale soil moisture observations, or when assimilating these observations in models, attention should be paid to the uncertainty that results from upscaling the point-scale observations to areal average soil moisture.

One of the first reports of soil moisture variability was made by Reynolds (1970). Other early reports on extensive studies of soil moisture variability were made by Bell et al. (1980) and Hawley et al. (1983). Several authors have noted that, despite the large spatial variability, the soil moisture patterns themselves remain relatively stable over time (e.g. Vachaud et al., 1985; Mohanty and Skaggs, 2001). This temporal persistence in the spatial pattern of soil moisture was

Published by Copernicus GmbH on behalf of the European Geosciences Union. 


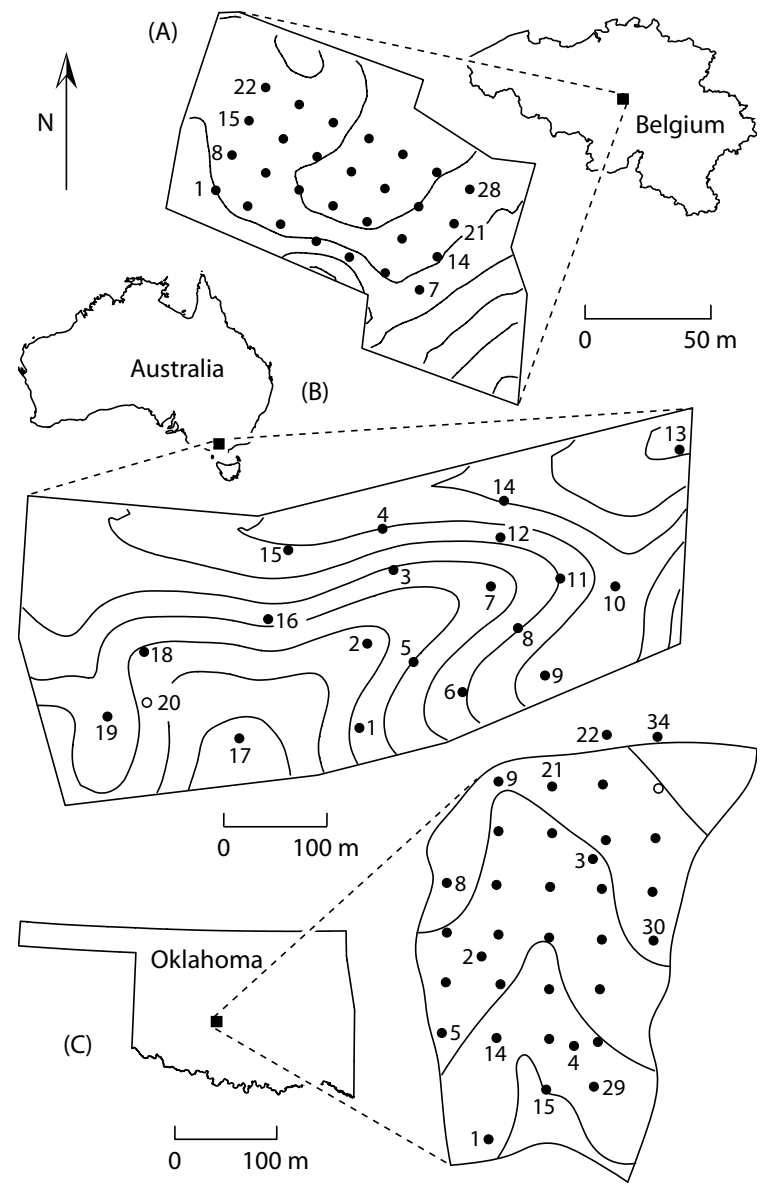

Fig. 1. Location of the study areas and observation sites. (a) Louvain-la-Neuve (0.5 m interval contour lines), (b) Tarrawarra ( $2 \mathrm{~m}$ interval contour lines), (c) R-5 ( $\sim 3 \mathrm{~m}$ interval contour lines). For Louvain-la-Neuve and R-5 the numbering of sites is continuous along rows. Open circles indicate sites that were omitted in the analysis.

used by Vachaud et al. (1985) to show that some sites maintain a similar rank throughout the year, i.e. that some sites are more representative of the spatial mean than others. Vachaud et al. (1985) called these sites "time stable". In this paper, we will use the term "rank" stability rather than "temporal" stability, following the arguments of Chen (2006). Rank stability has been used in many other studies to investigate spacetime dynamics of soil moisture fields and the potential of using a limited number of sites to observe the mean response (e.g. Kachanoski and de Jong, 1988; Comegna and Basile, 1994; Grayson and Western, 1998; Gómez-Plaza et al., 2000; Mohanty and Skaggs, 2001; Grant et al., 2004; Jacobs et al., 2004; Petrone et al., 2004; Martínez-Fernández and Ceballos, 2005). Pachepsky et al. (2005) reported rank stability to exist also in the vertical soil moisture distribution.

Several authors have reported that soil moisture patterns reflect patterns in vegetation (e.g. Hupet and Vanclooster, 2002; Schume et al., 2003; Hupet and Vanclooster, 2005), soil texture (e.g. Price and Bauer, 1984; Vachaud et al., 1985; Seyfried, 1998; Grant et al., 2004) and/or landscape characteristics (e.g. Anderson and Kneale, 1980; Nyberg, 1996; Crave and Gascuel-Odoux, 1997; Bárdossy and Lehmann, 1998; Famiglietti et al., 1998; Qiu et al., 2001). It is also known from field observations that soil moisture patterns can reflect patterns in landscape and/or soil characteristics in a wet state, while reflecting vegetation and/or soil characteristics in a dry state (Grayson et al., 1997; Fitzjohn et al., 1998; Gómez-Plaza et al., 2000). Recent advances in the theoretical understanding of these empiral findings have been made by Albertson and Montaldo (2003). They showed that the temporal evolution of soil moisture variability is driven by the sum of the covariances between soil moisture and different fluxes. In a simulation study, Teuling and Troch (2005) showed how the temporal dynamics of these different covariance terms can be used to explain the different observed relationships between mean soil moisture and its spatial variability. The fact that different controls operate on the spatial soil moisture pattern depending on the mean moisture content (e.g. Teuling and Troch, 2005; Wilson et al., 2005) has important implications for soil moisture sampling and the applicability of the rank stability concept. For instance, Martínez-Fernández and Ceballos (2005) concluded that it might take a year of sampling (a complete seasonal cycle) to correctly identify the most rank stable site.

Spatial soil moisture fields are known to exhibit a correlation structure. Western et al. (1998) provide an comprehensive table listing different geostatistical analyses of soil moisture fields. In general, correlation lengths for soil moisture are small, with values of only $10-25 \mathrm{~m}$ being common (Loague, 1992). Even in experiments with a relatively dense network, spatial correlation is often non-existent (Comegna and Basile, 1994; Hupet and Vanclooster, 2002). When terrain has a significant impact on the soil moisture pattern, the larger correlation lengths can mainly be attributed to terrain indexes (Western et al., 1998). In Tarrawarra, the spatial structure of the soil moisture field is known to be governed by topographic features in the wet state, while there is little spatial correlation in the dry state (Western et al., 1999).

In this paper we investigate the relationship between the dynamics of individual soil moisture observations and those of the spatial mean at the field- or small catchment scale. We perform this exercise for three different datasets, each having its own typical geographic and climatic conditions. By doing so, (dis)similarities between the datasets can be identified. Different sampling strategies are identified and methods are presented to quantify the corresponding uncertainties.

\section{Data}

The datasets were selected based on three criteria: a) observations on multiple depths so that a root-zone average soil moisture content can be estimated, b) a sufficient number of 
sites that allows for accurate estimation of the spatial mean root zone soil moisture content, and c) sufficient temporal dynamics so that the temporal variability over a complete seasonal cycle is well represented. For the depth of the root zone we used the upper $\sim 70 \mathrm{~cm}$ of the soil, which represents most (if not all) of the temporal dynamics of the soil moisture that is available for root water uptake. Typically, $50 \%$ of the roots are already located in the upper decimeters of soil (Schenk and Jackson, 2002). The depth is partly constrained by the observations, since we choose to include the observations made at $60 \mathrm{~cm}$ depth (see below). Furthermore we choose a constant depth for all datasets, so that the results can be compared directly. It should be noted that the temporal dynamics of soil moisture strongly decrease with depth, so that our results are not very sensitive to the exact depth over which soil moisture is averaged.

The 10.5 ha Tarrawarra catchment is located in southeastern Australia (Fig. 1b). The catchment has been subject to several intensive monitoring campaigns that aimed at investigating the soil moisture spatial pattern at the small catchment scale. The soil texture in the catchment varies from silty-loam to clay, and the topography is undulating with a maximum relief of $27 \mathrm{~m}$. The climate is temperate. Land use is perennial pastures used for grazing. At 59 dates between 20 September 1995 and 10 June 1997, soil moisture was monitored at 20 locations by means of a Neutron Moisture Meter (NMM). Observations were made at depths of 15, $30,45,60,90,120$, and $150 \mathrm{~cm}$, or to the depth of the access tube. Root zone soil moisture is taken as the average value of the observations at $15,30,45$, and $60 \mathrm{~cm}$ depth. Site 20 was excluded from the analysis since this data is suspected to be erroneous (Grayson and Western, 1998). Furthermore days with missing observations for one or more sites were excluded, leaving observations at 54 days at 19 sites. The time series of the spatial mean and standard deviation are shown in Fig. 2a. The NMM data was extracted from the Tarrawarra database (Western and Grayson, 1998).

The R-5 experimental catchment is located northeast of Chickasha, Oklahoma (USA). The USDA Agricultural Research Service intensively monitored R-5 from 1966 to 1978. The 10 ha catchment is a native grassland pasture used for grazing. The surface is gently sloping with an average slope of 3\% (Fig. 1c). At 84 dates between 21 January 1971 and 24 June 1974, NMM observations of soil moisture were made at 34 sites and at 8 different depths. Here we use the average value of the observations made at $15,30,45$, and $60 \mathrm{~cm}$ depth. One site (21) was excluded from the analysis since soil moisture at this site exhibited a suspicious drift, leaving observations at 84 days and 33 sites. The time series of the spatial mean and standard deviation are shown in Fig. 2b. The R-5 dataset is described in detail by Loague (1992).

Soil moisture variability was measured in an 0.65 ha agricultural field in Louvain-la-Neuve (Belgium) as part of a campaign that aimed at investigating the within-field spatial variability of evapotranspiration. Observations were made on
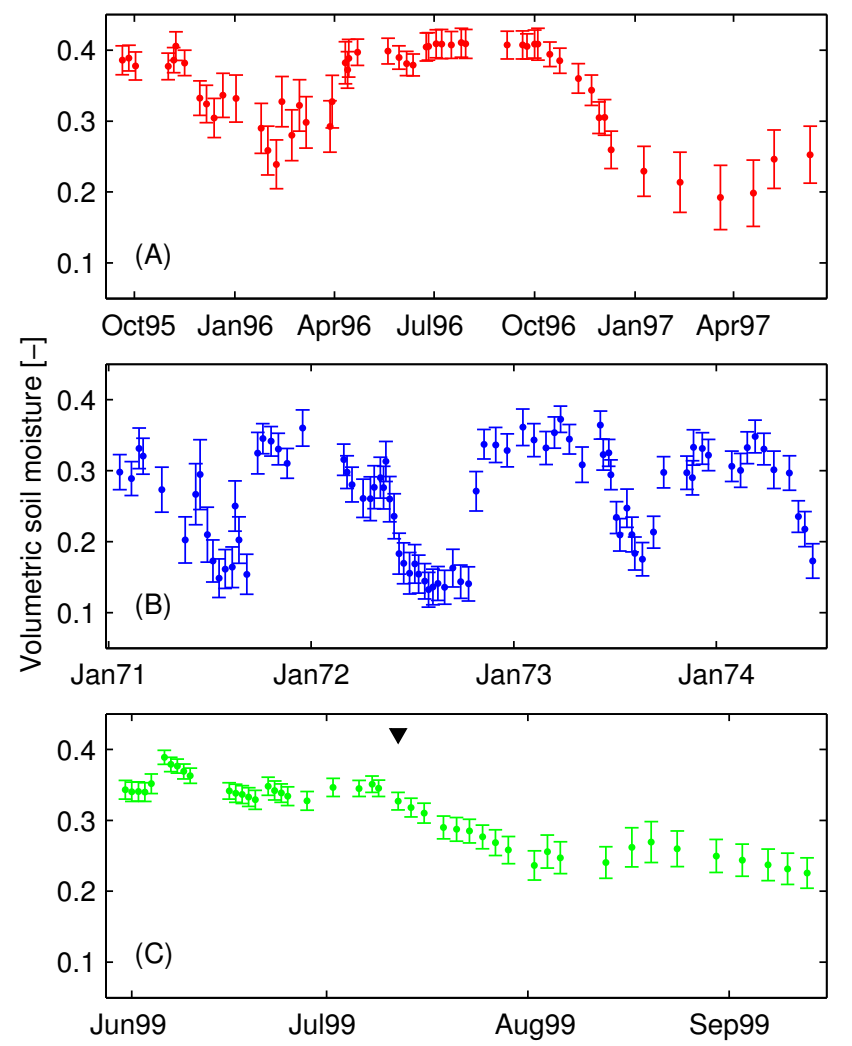

Fig. 2. Time series of spatial mean and variability. Error bars indicate \pm one standard deviation. (a) Tarrawarra (10.5 ha, 19 sites), (b) R-5 (10 ha, 33 sites), (c) Louvain-la-Neuve (0.65 ha, 28 sites). The downward triangle indicates the date of LAI observations.

45 days between 30 May 1999 and 13 September 1999. The observations were made on a regular $4 \times 7$ grid (see Fig. 1a) at different depths. He we use the average value of the Time Domain Reflectometry (TDR) observations $(0-20 \mathrm{~cm}$ ), and the NMM observations at depths of 25 and $50 \mathrm{~cm}$. The soil in the field is classified as well-drained silty-loam and there is little relief. During the campaign the field was cropped with maize. The climate is moderate humid. The time series of the spatial mean and standard deviation are shown in Fig. 2c. In this paper we also employ the Leaf Area Index (LAI) measurements that were taken at each site on 12 July 1999. The dataset is described in detail by Hupet and Vanclooster (2002).

\section{Method}

\subsection{Notation}

In this paper, we study aspects of the spatial and temporal dynamics of the volumetric soil moisture field $\theta(\boldsymbol{x}, \boldsymbol{t})$. Observations of this field are available at discrete intervals in space and time, namely $\boldsymbol{x}=\left\{x_{1}, x_{2}, \ldots, x_{n}\right\}$ and $\boldsymbol{t}=\left\{t_{1}, t_{2}, \ldots, t_{k}\right\}$ where $n$ and $k$ refer to the number of observation sites resp. 
Table 1. Summary of the observation strategies used in this paper. In the "Goal" column, "Abs" refers to absolute value of the spatial average volumetric soil moisture content, and "Dyn" refers to the dynamics of the spatial average.

\begin{tabular}{|c|c|c|c|}
\hline & Goal & $\begin{array}{l}\text { Number } \\
\text { of sites }\end{array}$ & Location of site(s) \\
\hline I & Abs & Single & Random \\
\hline II & Abs & Single & $\begin{array}{l}\text { On average closest } \\
\text { to spatial mean }\end{array}$ \\
\hline III & Abs & Single & Best regression with spatial mean \\
\hline IV & Abs & Single & $\begin{array}{l}\text { At single date closest } \\
\text { to spatial mean }\end{array}$ \\
\hline $\mathrm{V}$ & Abs & Multiple & Random \\
\hline VI & Dyn & Single & Random \\
\hline VII & Dyn & Single & $\begin{array}{l}\text { Smallest variability in } \\
\text { difference to spatial mean }\end{array}$ \\
\hline VIII & Dyn & Single & Smallest RMSE \\
\hline IX & Dyn & Multiple & Random \\
\hline
\end{tabular}

dates. The indexes $i$ and $j$ refer to selected locations in space and/or time. The number $m$ refers to the number of observations used in calculations when not all available observations are used $(m<n)$. We will analyse different strategies to estimate average soil moisture from point scale observations. Two main classes of strategies can be distinguished: those where the interest is in the soil moisture content itself (strategies I-V, see Sect. 3.3), and those where the interest is only in observing the dynamics of the spatial mean (strategies VIIX, see Sect. 3.4). An overview of these strategies is given in Table 1.

\subsection{Soil moisture distribution}

In this paper we will focus on the question if a set of soil moisture observations in space can, on average, be expected to follow a normal distribution. From other field experiments it is known that, while most sets are approximately normally distributed, individual sets of observations can show significant skewness and/or kurtosis (Famiglietti et al., 1998). Although there is no fundamental reason why soil moisture should follow a normal distribution, the use of this distribution has obvious advantages. Since soil moisture is bounded between residual moisture content and saturation, bounded distributions might be more appropriate for some applications (Wood, 1997; Ryu and Famiglietti, 2005).

Here we only perform a visual test for normality. For all $k$ observation dates, the individual samples $\theta\left(\boldsymbol{x}, t_{j}\right)$ are normalized by subtracting the observed spatial mean $\bar{\theta}_{j}$ and dividing by the observed standard deviation $s\left(\theta_{j}\right)$. These are estimated by:

$\hat{\mathrm{E}}\left[\theta\left(t_{j}\right)\right]=\bar{\theta}_{j}=\frac{1}{n} \sum_{i=1}^{n} \theta\left(x_{i}, t_{j}\right)$ and

$\widehat{\operatorname{Var}}\left[\theta\left(t_{j}\right)\right]=s\left(\theta_{j}\right)^{2}=\frac{1}{n-1} \sum_{i=1}^{n}\left[\theta\left(x_{i}, t_{j}\right)-\bar{\theta}_{j}\right]^{2}$

These normalized values are then ranked from dry to wet and plotted with their corresponding cumulative probability level. The probability axis is transformed such that a normal distribution yields a straight line. Furthermore the relation between the mean soil moisture content and the standard deviation is investigated.

\subsection{Mean soil moisture estimation}

In practice, the spatial average soil moisture content is often assumed to equal that at a single observation site (strategy I). For this strategy, the uncertainty of this estimate is controlled by the spatial variability. This spatial variability might depend on the mean moisture content, which is generally unknown (since this is to be estimated). Therefore we define the "expected" spatial variability for each dataset, i.e. the variance that can be expected at a given moment in time without prior knowledge of $\bar{\theta}_{j}$ (see Appendix A):

$\mathrm{E}[\operatorname{Var}(\theta)]=\frac{1}{k} \sum_{j=1}^{k} \operatorname{Var}\left[\theta\left(t_{j}\right)\right]$

With the a priori knowledge that soil moisture patterns are persistent, some sites are more representative of the spatial mean than others. Following Vachaud et al. (1985), we define the spatial difference $\delta\left(\boldsymbol{x}, t_{j}\right)$ between the soil moisture content $\theta\left(\boldsymbol{x}, t_{j}\right)$ and the spatial mean water content $\bar{\theta}_{j}$ as:

$\delta\left(\boldsymbol{x}, t_{j}\right)=\theta\left(\boldsymbol{x}, t_{j}\right)-\bar{\theta}_{j}$

In contrast to previous studies on rank stability where $\delta$ was normalized by $\bar{\theta}_{j}$, we express $\delta$ in the same units as $\theta$. Note that since $\delta$ is corrected for the spatial mean soil moisture at all $t_{j}$, it reflects the persistence in the spatial pattern of $\theta$ rather than in its actual magnitude. The temporal mean difference for every site $\bar{\delta}_{i}$ is estimated as:

$\hat{\mathrm{E}}\left[\delta\left(x_{i}\right)\right]=\bar{\delta}_{i}=\frac{1}{k} \sum_{j=1}^{k} \delta\left(x_{i}, t_{j}\right)$

and the temporal variability of $\delta$ at site $i, \widehat{\operatorname{Var}}\left[\delta\left(x_{i}\right)\right]$, as:

$\widehat{\operatorname{Var}}\left[\delta\left(x_{i}\right)\right]=s\left(\delta_{i}\right)^{2}=\frac{1}{k-1} \sum_{j=1}^{k}\left(\delta\left(x_{i}, t_{j}\right)-\bar{\delta}_{i}\right)^{2}$

Although various definitions can be found in the literature for the most rank stable site $\Phi$, the most straightforward definition is the site having the smallest absolute mean difference so that this site can be used directly to estimate the mean soil moisture content (e.g. Grayson and Western, 1998):

$\Phi=\left\{i \quad|\quad| \bar{\delta}_{i}|<| \bar{\delta}_{m} \mid \quad \forall \quad m \neq i\right\}$ 
Through this definition, the most rank stable site can only be selected if the spatial and temporal dynamics of the soil moisture field are known. This applies to cases where an area was first subjected to an intensive monitoring campaign, and where monitoring is continued only at the site which, on average, is closest to the spatial mean (strategy II). For strategy II we will assume $\mathrm{E}\left(\bar{\delta}_{\Phi}\right)=0$.

If the space-time variability of a soil moisture field is known from a preceding campaign, the spatial mean might also be predicted from a regression between soil moisture at an individual site and the spatial mean. Since the soil moisture differences at individual locations might be a function of $\bar{\theta}_{j}$, this strategy can be expected to yield more accurate estimates of $\bar{\theta}_{j}$ than strategy II. Since in general the best site will be chosen, we quantify the uncertainty associated with this approach as the minimum of the variances around the regressions for the individual sites (strategy III).

A serious drawback for application of strategy II is that a priori knowledge on the space-time dynamics of the soil moisture field is required. This requires intensive sampling. As was suggested in previous studies (e.g. Vachaud et al., 1985; Comegna and Basile, 1994), a more practical method would be to select the most rank stable site from one initial field survey at time $t_{j}$. With this strategy (strategy IV), the most rank stable site $\Phi_{j}$ is the one that is closest to $\bar{\theta}_{j}$. We therefore determine $\Phi_{j}$ for all $k$ spatial soil moisture fields. The effective uncertainty associated with the approach of taking $\Phi_{j}$ to represent $\bar{\theta}_{j}$ is influenced both by $\bar{\delta}_{i}$ and $\operatorname{Var}\left[\delta\left(x_{i}\right)\right]$. The expression for this "overall" variance is derived in Appendix A.

If the mean soil moisture can be estimated from multiple measurements located randomly (strategy V), the uncertainty of the mean will decrease with the number of observations $m$. Whereas in previous studies the focus was mainly on the relative accuracy of the mean estimated from multiple observations (or the numbers of observations needed to achieve a required level of relative accuracy), we focus here on the absolute uncertainty since this is a more relevant parameter for many modeling purposes. In the idealized case where the observations are completely independent, the standard error of the mean $s_{m}$ is given by:

$s_{m}=\frac{s}{\sqrt{m}}$

where $s$ is the standard deviation of the individual observations. Since the soil moisture field exhibits spatial correlation, the actual dependence of $s_{m}$ on the number of observations will differ from Eq. (8). To investigate whether the actual dependence differs from Eq. (8), we derived this dependence from the observations. For all observation dates and for $1 \leq m \leq n / 2$, the spatial mean was estimated for all independent sets of observations of size $m$. These sets of observations were selected randomly, but every site was only allowed to occur once, yielding a maximum number of $n / m$ sets. The standard error of the mean was then calculated as the standard deviation of the estimated means. This procedure was repeated 20 times to reduce sampling effects, and the results were averaged.

\subsection{Mean soil moisture time series estimation}

One might argue that for a particular site $i$ the bias with respect to the spatial mean $\left(\bar{\delta}_{i}\right)$ is of little importance as long as the dynamics of the spatial mean are well represented. In this case the goal of soil moisture monitoring might be to estimate the spatial mean soil moisture dynamics rather than its actual value. The variability of the difference between a time series at one site and the time series of the spatial mean is expressed by $\operatorname{Var}\left[\delta\left(x_{i}\right)\right]$. This quantity expresses the temporal variability of a site with respect to the spatial mean, similar to $\operatorname{Var}\left[\theta\left(t_{j}\right)\right]$ for the spatial mean. If a site is randomly selected (strategy VI), the "expected" temporal variance with respect to the spatial mean is (see Appendix A):

$\mathrm{E}\left\{\operatorname{Var}\left[\delta\left(x_{i}\right)-\bar{\delta}_{i}\right]\right\}=\frac{1}{m} \sum_{j=1}^{m} \operatorname{Var}\left[\delta\left(x_{i}, t_{j}\right)\right]$

If the goal is to capture the temporal dynamics of the spatial mean from a single observation site, a different definition of rank stability might be more appropriate. In this case (strategy VII) one would prefer the site with the smallest temporal variance of $\delta$ :

$\Phi=\left\{i \quad \mid s\left(\delta_{i}\right)^{2}<s\left(\delta_{m}\right)^{2} \quad \forall \quad m \neq i\right\}$

Note that through this definition, the most rank stable site can only be identified if the complete space-time dynamics are known. This is different from strategy IV, where only one spatial field is needed to identify the site closest to the mean. Other definitions for the most rank stable site can also be found. For instance, Jacobs et al. (2004) account for both the bias and variance of the soil moisture difference time series in the definition of the most rank stable site by minimizing the root mean square error:

$\operatorname{RMSE}_{i}=\left\{\bar{\delta}_{i}^{2}+\operatorname{Var}\left[\delta\left(x_{i}, \boldsymbol{t}\right)\right]\right\}^{\frac{1}{2}}=\mathrm{E}\left\{\left[\delta\left(x_{i}\right)\right]^{2}\right\}^{\frac{1}{2}}$

This case (strategy VIII) will be analyzed in addition to the other definitions of rank stable sites. As was already mentioned in the preceding paragraph, the soil moisture differences might depend on $\bar{\theta}_{j}$. This means that a better estimate of the mean response can be obtained by regression of soil moisture at an individual site to the spatial mean. Since uncertainty around a regression is not affected by bias, this is the same as strategy III. The uncertainty in the soil moisture time series at a single site with respect to the spatial mean time series might be reduced by taking the time series of soil moisture averaged over different randomly located sites (strategy IX). To quantify this reduction, we used an approach similar to that for strategy V. For all observation dates and for $1 \leq m \leq n / 2$, the spatial mean was estimated for 


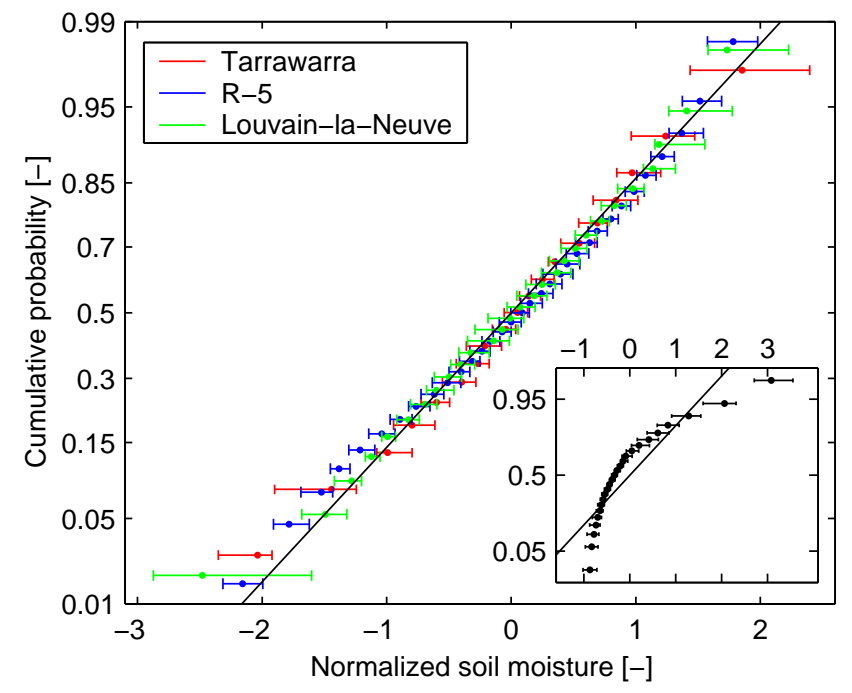

Fig. 3. Normal probability plot of the normalized spatial soil moisture fields. Dots indicate the median value, error bars indicate $25 \%$ and $75 \%$ percentiles of time variability. The inset shows the same procedure applied to randomly generated fields from a lognormal distribution, with $n=25$ and $k=60$.

all independent sets of observations of size $m$. The uncertainty was then calculated as the variability in the difference between the time series of these estimates and the time series of the "true" spatial mean.

\subsection{A priori site selection}

Important advances can be made in the observation of mean soil moisture if the sites that best represent the mean conditions can be identified a priori; i.e. if the location of these sites can be explained by a land surface property that is known to influence soil moisture dynamics (soil texture, vegetation). For instance, Vachaud et al. (1985) already discussed the relation between rank stability and soil texture. They stated that "there is a high probability that if a location is the most wet at a given time, it will remain the most wet at other times because it has the highest clay content". For more hilly areas, Grayson and Western (1998) argued that these sites "are in areas that are neither strongly convergent nor divergent, tend to be near the mid-slopes and are in areas that have topographic aspect close to average for the catchment". Detailed observations of land surface properties are often not available at the exact location of the soil moisture sites. A detailed $5 \times 5 \mathrm{~m}$ Digital Elevation Model is available for the Tarrawarra catchment (Western and Grayson, 1998). For the Louvain-la-Neuve, observations of Leaf Area Index (LAI) at all 28 soil moisture sites are available (Hupet and Vanclooster, 2002).

Terrain is known to influence the spatial distribution of soil moisture. Many different wetness indexes found in the literature predict zones of below/above average wetness based on topography (as represented by a Digital Elevation Model, DEM). Several of these indexes were employed by Western et al. (1999) to study the degree in which they can be predict the spatial organization of soil moisture at Tarrawarra. In this study we employ a wetness index developed by Svetlitchnyi et al. (2003). The (semi-)empirical model accounts for the effects of slope profile shape, slope aspect, distance from the divide, and slope gradient on the soil moisture distribution in the top $0.5 \mathrm{~m}$ of the soil (Svetlitchnyi et al., 2003). In this way both effects of subsurface flow and exposure are accounted for. The relative wetness coefficient at any point is defined as the ratio of expected soil moisture at that point (as influenced by topography alone) and the expected soil moisture for a flat surface (without effects of topography). Details of the model and how it can be derived from a DEM can be found in Svetlitchnyi et al. (2003). Following this approach, sites that accurately represent the dynamics of the spatial mean should have a wetness coefficient near unity. Here we investigate the correlation between the wetness coefficient and the mean soil moisture difference $\bar{\delta}_{i}$.

Leaf area index is known to influence evapotranspiration: higher LAI leads to higher evapotranspiration rates (e.g. AlKaisi et al., 1989; Hupet and Vanclooster, 2004). This means that sites with higher than average LAI will have evaporated more in the preceding period. At this site, one should expect a below-average soil moisture. In this way, a site with average LAI might be associated with average evapotranspiration rates, and average soil moisture. In this paper, we investigate the correlation between LAI and the mean soil moisture difference $\bar{\delta}_{i}$.

\section{Results}

\subsection{Soil moisture distribution}

Figure 3 shows the normalized soil moisture versus the cumulative probability for the three datasets. The straight line indicates the standard normal distribution. In this plot, any structural deviation from a normal distribution will result in a deviation from the straight line. From the scatter in the points (indicated by error bars) it can be seen that individual distributions can deviate significantly from normal. However on average the points tend to cluster around the normal line. This shows that, if no a priori information on the spatial soil moisture distribution is available, the assumption of normality is reasonable. R-5 shows the least temporal variability in the shape of the soil moisture distribution. These findings are in agreement with previous studies (Cosh et al., 2004).

To illustrate that the clustering around the straight line is not the result of sampling or the Central Limit Theorem, we performed the same analysis on randomly generated spatial fields drawn from a lognormal distribution. The inset in Fig. 3 shows that sampling has a minor impact on the results. 
Table 2. Effective uncertainties for the different sampling strategies. Values are expressed as one standard deviation (volumetric moisture content).

\begin{tabular}{llll}
\hline Strategy & Tarrawarra & R-5 & $\begin{array}{l}\text { Louvain- } \\
\text { la-Neuve }\end{array}$ \\
\hline Climate & 0.0639 & 0.0726 & 0.0471 \\
\hline I & 0.0274 & 0.0263 & 0.0167 \\
II & 0.0079 & 0.0122 & 0.0067 \\
III & 0.0014 & 0.0055 & 0.0025 \\
IV & 0.0166 & 0.0187 & 0.0150 \\
V & $0.0128^{*}$ & $0.0128^{*}$ & $0.0083^{*}$ \\
& $0.0072^{* *}$ & $0.0078^{* *}$ & $0.0048^{* *}$ \\
VI & 0.0162 & 0.0163 & 0.0115 \\
VII & 0.0076 & 0.0088 & 0.0042 \\
VIII & 0.0079 & 0.0099 & 0.0067 \\
IX & $0.0075^{*}$ & $0.0077^{*}$ & $0.0054^{*}$ \\
& $0.0042^{* *}$ & $0.0045^{* *}$ & $0.0031^{* *}$ \\
\hline
\end{tabular}

*Estimated uncertainty for 4 randomly located sites.

**Estimated uncertainty for 9 randomly located sites.

The randomly generated data from a non-normal distribution clearly deviates from a straight line.

It is well known from numerous field- and theoretical studies that spatial soil moisture variability might vary with the mean soil moisture content (for an overview, see Famiglietti et al., 1998). Figure 2 shows the observed mean and standard deviation of the spatial soil moisture fields. The standard deviation ranges from 0.01 to 0.05 , while most values are between 0.02 and 0.03 . All three datasets show an increase in variability with decreasing moisture content, with Tarrawarra showing the strongest trend and R-5 the weakest. Louvain-la-Neuve exhibits the lowest variability. This is likely due to the smaller size of the area (more than an order of magnitude), the little variation in topography and/or soils as compared to the other sites.

\subsection{Mean soil moisture estimation}

With only one observation site located randomly (strategy I), the uncertainty associated with estimates of the mean soil moisture is controlled by the spatial variability. Effective variability values are listed in Table 2 . Tarrawarra and R5 have comparable standard deviations of 0.027 and 0.026 , while Louvain-la-Neuve has a somewhat smaller value of 0.017. Since the spatial distribution of soil moisture is approximately normal, $95 \%$ confidence intervals can be constructed with a width of twice the expected spatial standard deviation. It is interesting to compare these values to variability in the climate signal itself, that is the temporal variability of the spatial mean $\bar{\theta}_{j}$ (see Fig. 2). Since this is the signal that one actually wants to observe, a comparison with the uncertainty (or noise) provides something that can be in-
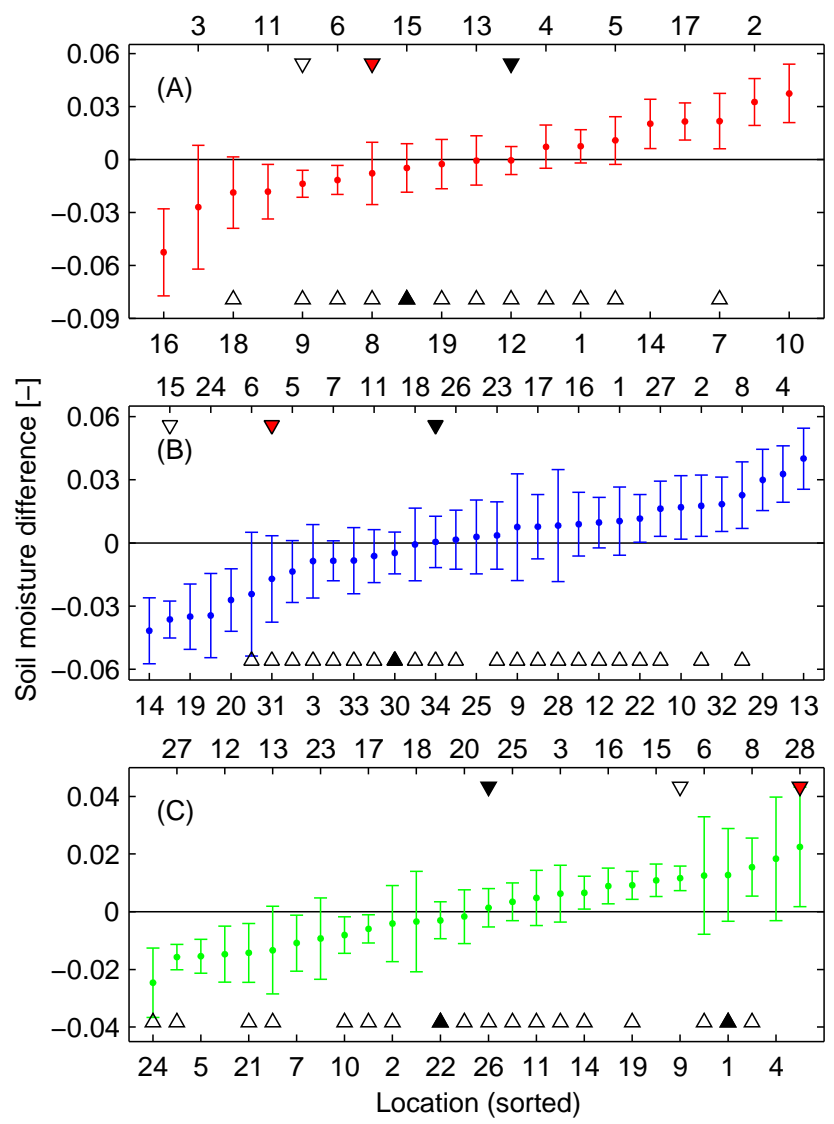

Fig. 4. Rank stability plots for the three datasets. The sites have been ranked according to their mean difference with the spatial mean. The error bars indicate temporal variability (standard deviation). The numbers refer to sites in Fig. 1. (a) Tarrawarra, (b) R-5, (c) Louvain-la-Neuve. Downward triangles at the top indicate the most rank stable site (filled for strategy II, open for strategy VII, red for strategy III, green for strategy VIII), upward triangles at the bottom indicate most rank stable sites for strategy III with the most probable one(s) filled.

terpreted as a signal-to-noise ratio. The variability of the climate signal is also listed in Table 2.

A much more precise estimate of the mean soil moisture from a single site can be obtained if this site is on average closest to the spatial mean (strategy II). These sites are indicated in Fig. 4 by the downward triangles. With this strategy the uncertainty reduces to the temporal variability of the difference between soil moisture at this site and the spatial mean. These values vary between 0.007 and 0.012 (see Table 2).

Even more precise estimates of the mean soil moisture from a single site are obtained with a linear regression of soil moisture at any site with the mean (strategy III). Table 2 lists the minimum values of this observation strategy. The corresponding sites are identified in Fig. 4. For all three datasets, these sites differ from the sites that are on average closest 


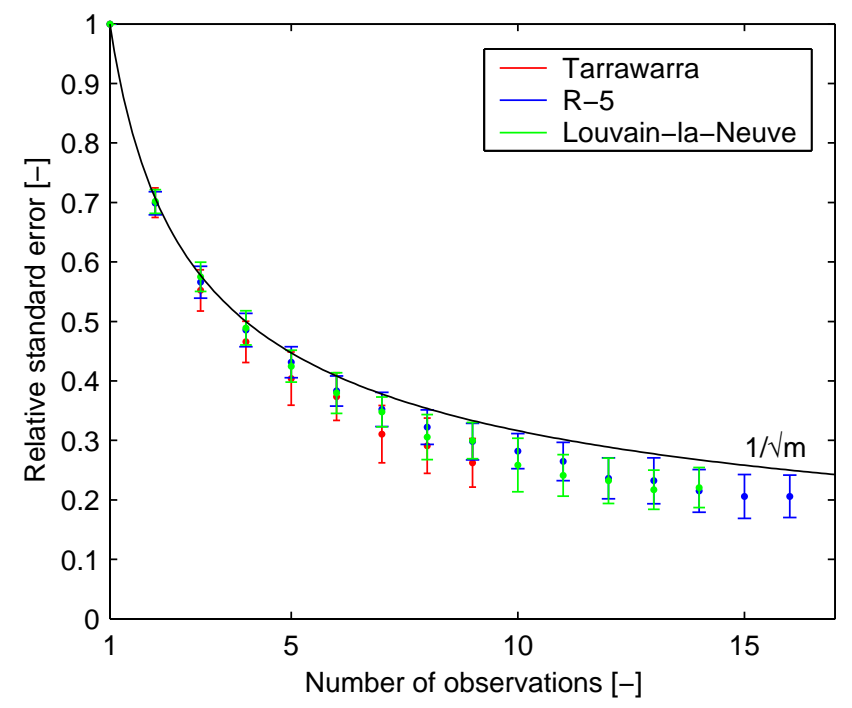

Fig. 5. Relative standard error as a function of the number of observations that is used to estimate the spatial mean. The black line is the theoretical curve for independent observations.

to the mean. These sites also have a large time variability, indicating that the corresponding regression not only has an offset, but also that the slope differs from 1 . The low uncertainty (0.0014 to 0.0055$)$ indicates that accurate soil moisture estimation from a single site is possible over a range of wetness conditions; but only if the space-time dynamics of the soil moisture field are known from a preceding campaign.

As noted before, the site closest to the mean might in practice be identified from a single spatial survey (strategy IV). If there would exist perfect rank stability, this would give the same result as strategy II. However careful analysis reveals that the site that is on average closest to the mean has a low probability of being identified at a given moment in time. These probabilities are only 7\% (4/54), 10\% (8/84), and $4 \%(2 / 45)$ for Tarrawarra, R-5, and Louvain-la-Neuve, respectively. On individual dates, between 60 to $70 \%$ of all the sites would be identified as being closest to the spatial mean. These sites are identified in Fig. 4 by the upward triangles. The site(s) that is (are) most likely to be identified as being closest to the mean on individual dates (indicated by filled upward triangles) differ in all three cases from the site that is on average closest to the mean. For Tarrawarra, R-5, and Louvain-la-Neuve these sites are resp. 15, 30, and $1(22)$, with probabilities of $19 \%$ (10/54), $14 \%(12 / 84)$, and $11 \%(5 / 45)$.

The large variation in sites being closest to the mean on individual dates adds considerable uncertainty to strategy II. For Louvain-la-Neuve, this uncertainty is almost equal to the spatial variability $(0.0150$ vs. 0.0167$)$. This is caused by the selection of some sites with a large temporal variability of the soil moisture difference. For the other two datasets, the uncertainty for strategy IV is still $61 \%$ (Tarrawarra) resp. $71 \%$ (R-5) of the effective spatial standard deviation.
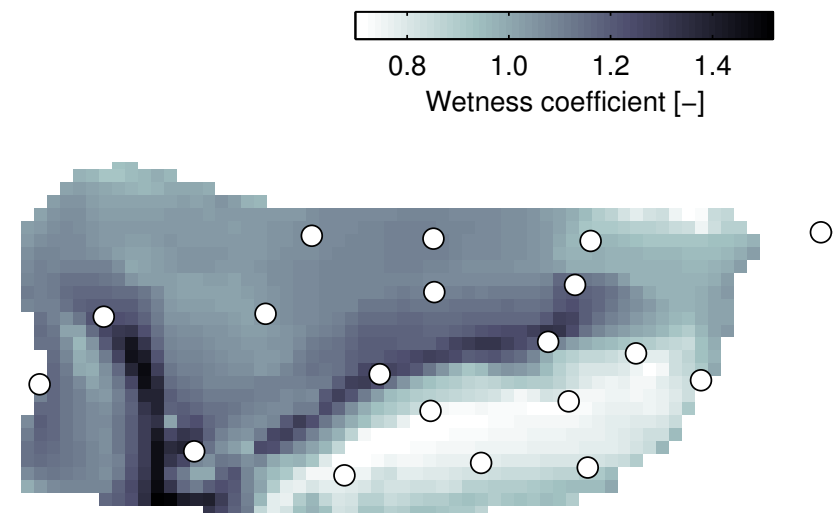

Fig. 6. Distribution of the wetness coefficient for the Tarrawarra catchment. Circles indicate the soil moisture sites.

The uncertainty of the estimated mean from point-scale observations is reduced if the mean can be estimated from multiple observations. Figure 5 shows how the relative (to the spatial variability) uncertainty decreases with the number of randomly located observation sites. In spite of any possible spatial correlation, the empirical relationships for the different datasets are close to the theoretical relation for fully independent samples $(1 / \sqrt{ } m)$. For all datasets, this relation slightly overestimates the actual uncertainty. When the mean of 4 independent samples is used to estimated the "true" spatial mean, the uncertainty reduces to approximately $50 \%$ of the spatial variability. For 9 sites, this reduction is 70 to $75 \%$. For independent samples, this reduction should be $50 \%$ for four samples, and $66.7 \%$ for nine samples.

\subsection{Mean soil moisture time series estimation}

One might expect the uncertainty associated with the estimation of the temporal dynamics of the spatial mean from a single site to be much less than that associated with the estimation of the mean itself. The effective values of the uncertainty on the time series range between 0.0115 and 0.0163 (see Table 2). It is interesting to compare the values in Table 2 for strategies I and VI. Strategy I represents the effective (for all sampling dates) uncertainty caused by spatial heterogeneity, while strategy VI represents the effective (for all sampling sites) uncertainty due to temporal or process heterogeneity. The ratios of the temporal and spatial standard deviation around the spatial mean range between 0.59 and 0.68 . While the spatial variability is known to be large, little attention has been paid so far in soil moisture research to temporal variability with respect to the spatial mean.

There are large differences in the temporal variability between the different sites. For some sites, the temporal variability exceeds the spatial variability, while other sites show little temporal variability in their difference to the spatial mean. The sites with minimum temporal variability are indicated in Fig. 4. In all the three datasets, these sites do not 


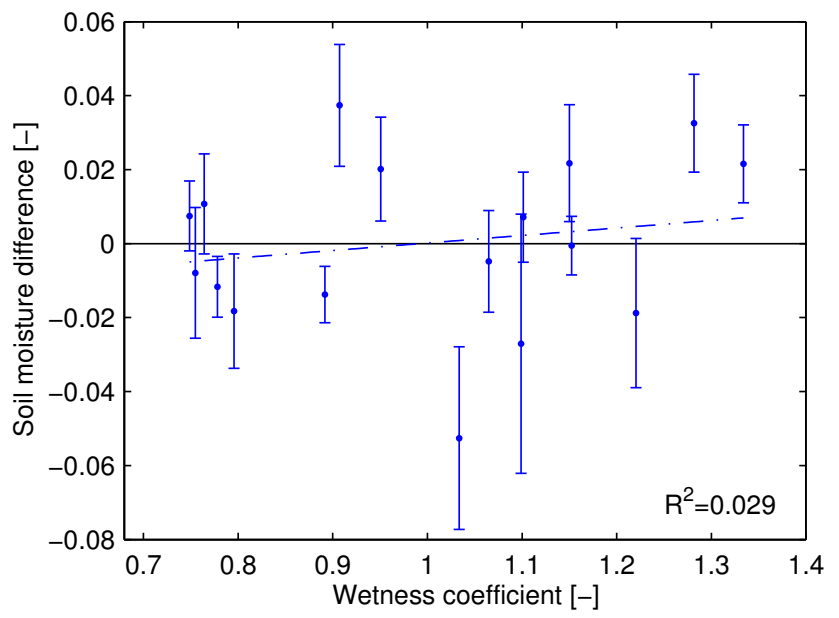

Fig. 7. Wetness coefficient versus the mean soil moisture difference for all sites at Tarrawarra. Error bars indicate the temporal variability in soil moisture difference for each site, similar to Fig. 4.

correspond to the optimal sites for the other strategies. In two datasets (R-5 and Louvain-la-Neuve), these sites even differ from all the sites that are closest to the spatial mean at individual dates. The uncertainty for these sites is small: between 0.0042 and 0.0088 . However even for the "best" site, the standard deviation is still $10 \%$ of the climate signal.

The site with the lowest RMSE is indicated in Fig. 4. In two datasets (Tarrawarra and Louvain-la-Neuve) the site with the lowest RMSE is on average also closest to the spatial mean, showing that in practice there might be little difference between the definitions. The uncertainties for strategy VIII (0.0053 to 0.0080) are comparable to the values for strategies II and VII.

If the time series of the spatial mean is estimated from the time series of the mean of several randomly located sites (strategy IX), then the relative uncertainty (with respect to the "expected" temporal variability) decreases with the number of sites in a similar fashion to Fig. 5 (not shown). Table 2 lists the uncertainties in the case that four resp. nine sites are used to estimate the spatial mean. These values are roughly $45 \%$ resp. 25 to $30 \%$ of the variability in the individual time series (strategy VI), which again is only slightly less than would follow from Eq. (8).

\subsection{A priori site selection}

Figure 6 shows the distribution of the wetness coefficient for the Tarrawarra catchment. This coefficient is indicative for the distribution of soil moisture in the upper $0.5 \mathrm{~m}$ of the soil (Svetlitchnyi et al., 2003). The distribution of the wetness coefficient closely resembles the observed detailed soil moisture patterns at Tarrawarra (e.g. Western and Grayson, 1998). Above average wetness is encountered along the drainage lines, and below average wetness on the exposed north-facing slope. If point-scale soil moisture is only dis-

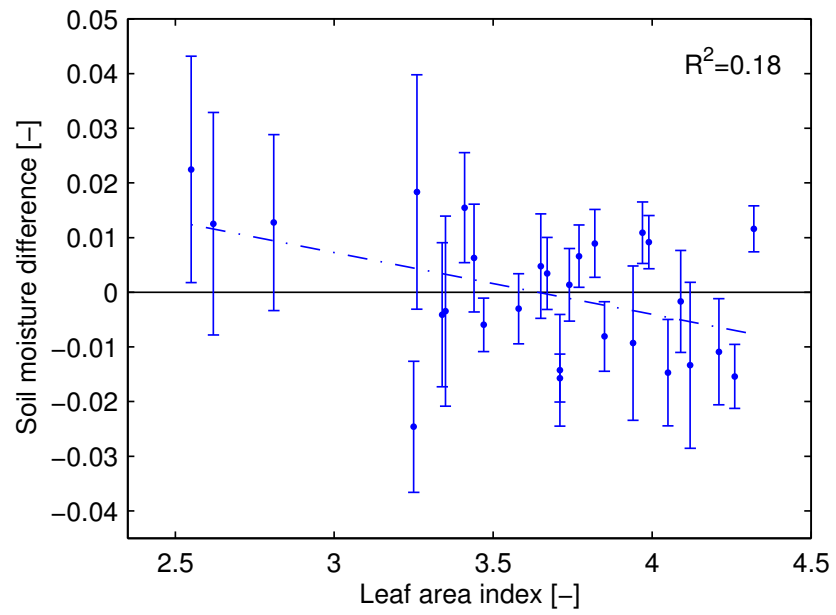

Fig. 8. Leaf area index versus the mean soil moisture difference for all sites at Louvain-la-Neuve. Error bars indicate the temporal variability in soil moisture difference for each site, similar to Fig. 4.

tributed according to topography, one might expect a clear relation with the wetness coefficient. Ideally, the sites that are closest to the spatial mean would have a wetness coefficient near unity. However the actual correlation between the mean soil moisture difference $\bar{\delta}_{i}$ and the wetness coefficient (Fig. 7) is close to zero. The low correlation is illustrated by the fact that the site which has a wetness coefficient closest to unity is on average the driest! This result is both surprising and contradicting: while the pattern of the wetness coefficient seems very similar to detailed observed soil moisture patterns at Tarrawarra, there is hardly any correlation between the individual NMM sites and the wetness coefficient. This is in line with the findings by Wilson et al. (2004), who showed that even in catchments with significant topographic variability, the topographic component might not be the largest contributor to the overall spatial variance.

For Louvain-la-Neuve, vegetation rather than topography can be expected to have the largest impact on the spatial soil moisture pattern. Figure 8 shows the relation between LAI and the mean soil moisture difference $\bar{\delta}_{i}$ for each site. Although the correlation is higher than for the wetness coefficient at Tarrawarra, it is still low $\left(R^{2}=0.18\right)$. This might be due to the fact that LAI was observed only once under relatively wet soil moisture conditions early in the growing season. The soil moisture pattern under these conditions might reflect the pattern of soil texture rather than vegetation (LAI). However the correlation with the yield, which was measured at the end of the growing season (Hupet and Vanclooster, 2002), is even lower $\left(R^{2}=0.16\right)$. These correlations are too weak to predict the location of rank stable sites from LAI alone. 


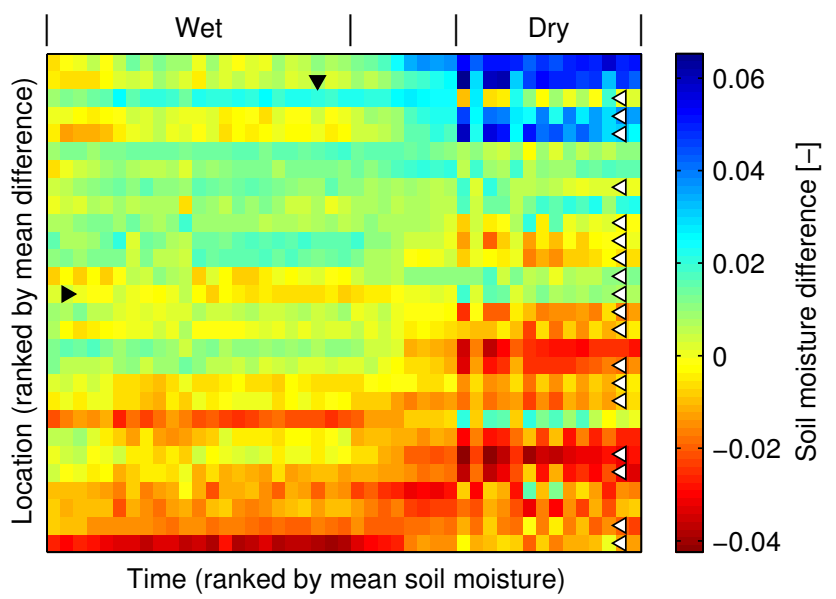

Fig. 9. Differences between local soil moisture and the spatial mean $(\delta(\boldsymbol{x}, t))$ for Louvain-la-Neuve. The sites have been ranked according to their mean difference (the same as in Fig. 4), and the dates according to their mean soil moisture content. Three domains can be distinguished with corresponding spatial patterns: a wet domain in which soil moisture variability reflects soil properties, a transition domain, and a dry domain in which the variability reflects vegetation properties. The leftward triangle indicates the site that is on average closest to the mean (strategy II), rightward triangles indicate sites that on single dates are closest to the mean, and the downward triangle indicates the date of LAI observations.

\section{Discussion}

The results presented in this paper are based on analysis of three datasets only. The large consistency in the relative uncertainties between the different observation strategies (Table 2) suggest they might be indicative for other areas as well.

So far, the local soil moisture differences $\delta_{i}$ to the spatial mean have only been discussed in terms of their expected distribution. It is also interesting to look at their temporal correlation. Figure 9 shows the space-time distribution of $\delta(\boldsymbol{x}, \boldsymbol{t})$ for Louvain-la-Neuve sorted by the temporal mean difference for every site and the spatial mean soil moisture for every date. This reveals an interesting property of the soil moisture field. For a large part of the mean soil moisture range (indicated by "wet"), the local soil moisture differences remain nearly constant, indicating a similar spatial pattern. Similarly, another (different!) spatial pattern exists in the "dry" domain, with a less defined transition in between. These patterns likely reflect properties of the soil in the "wet" domain, and vegetation in the "dry" domain. The apparent switch between two preferred spatial patterns is similar to the one observed in Tarrawarra (Grayson et al., 1997). It should be noted that the LAI observations were made in the "wet" domain, while the impact on the soil moisture pattern might be more pronounced in the "dry" domain. This might partly explain the low correlation in Fig. 8.
An interesting question not yet discussed in this paper is what is the uncertainty introduced by measurement error? From a geostatistical analysis of Tarrawarra TDR soil moisture, Western et al. (1998) report nugget values ranging from 0.020 to 0.024 (standard deviation) including both effects of small scale heterogeneity and measurement error. Western and Grayson (1998) report the NMM observations to have an error standard deviation of 2.5 volumetric moisture percent, i.e. 0.025 . These values seem to overestimate the actual random error. Table 2 shows that the scatter around the regression between soil moisture at individual sites and the spatial mean (strategy III) can be as low as 0.0014 . This is probably a better estimate of the true random error. For Louvain-la-Neuve, Hupet et al. (2004) report total measurement uncertainties for the NMM and TDR of 0.0091 to 0.0095 resp. 0.0158 to 0.0176 (standard deviation of the volumetric moisture content). The contribution of the instrument alone is estimated as 0.005801 resp. 0.0021 to 0.0028 . This is very close to the value for strategy III in Table $2(0.0025)$. This suggests that it is possible to monitor spatial average soil moisture with approximately the same accuracy as point-scale soil moisture, provided that the regression between the two is known.

The relative uncertainties can be used to make a first-order cost-benefit analysis when planning a field campaign. Depending on the length of the campaign, a trade off can be made between the costs associated with continuous monitoring at multiple randomly located sites (depending on the accuracy that is required), and monitoring at a single site that is known to yield accurate estimates of average soil moisture from initial extensive spatial variability surveys. Depending on the observation strategy that is chosen, a higher accuracy might be achieved at a lower cost. In terms of accuracy/cost ratio, it might be more beneficial to estimate mean soil moisture from several randomly located sites during shorter campaigns, while for long-term monitoring it might be better to establish a regression between the mean and the value at a single site that is to be monitored over several years. Preferably, this regression should at least contain two points in the dry and wet extremes.

The uncertainties in Table 2 can also be used to construct confidence bounds on the observations. These uncertainty bounds can be used to make more quantitative statements on how good model soil moisture (i.e. often with effective fieldor catchment-scale parameters) has to match with point-scale observations. Figure 10 shows an example of this for a wetdry transition in the R-5 dataset. The outer bounds in Fig. 10 correspond to strategy I when no information is available on how this site relates to the spatial mean. The middle bounds correspond to the uncertainty on the dynamics alone (i.e. strategy VI) when the site is located randomly. The inner bounds represents the uncertainty of the best-case scenario were the spatial average soil moisture is estimated from a known regression with the mean. 


\section{Conclusions}

- It is shown that, on average, the spatial distribution of soil moisture is well approximated by a normal distribution. This is true for all the three datasets. This property can be used to construct confidence intervals on pointscale soil moisture observations.

- The temporal dynamics of the spatial mean soil moisture can be estimated more accurately from a randomly located site than the mean soil moisture itself. However the standard deviation of the uncertainty on these temporal dynamics is still $\sim 66 \%$ of the effective spatial variability.

- Rank stable sites exist for all three datasets. The uncertainty on the estimated spatial mean is reduced considerably (to $\sim 40 \%$ of the effective spatial variability) if one of these sites is used to monitor soil moisture. However identification of these sites requires intensive sampling. If such a site is selected from a single spatial survey, the overall uncertainty is still $\sim 75 \%$ of the effective spatial variability.

- For many sites, the temporal correlation in the soil moisture differences to the spatial mean results in an accurate linear regression between soil moisture at that site and the spatial mean. The accuracy of this regression is close to the random observation error for a single soil moisture observation.

- To first order, the relative (to the spatial variability) standard error of the spatial mean soil moisture reduces with the inverse of the square root of the number of randomly located sites used to estimate the mean. This means that the uncertainty can be reduced by $\sim 50 \%$ if the mean is estimated from four sites rather than from a single site.

- Although the spatial soil moisture pattern is known to be related to a combination of soil, vegetation, and landscape characteristics, neither a wetness coefficient derived from a DEM or the LAI showed a high correlation with the temporal mean soil moisture differences to the spatial mean for the different sites. It should therefore not be expected that a particular site with an average wetness coefficient or average LAI has a close to average soil moisture.

\section{Appendix A}

Here, an expression is derived for the mean and variance of a random selection from $n$ densities of the same random variable $X, f_{i}(X)$, with $i=\{1,2, \ldots, n\}$. Each density $f_{i}(X)$ has

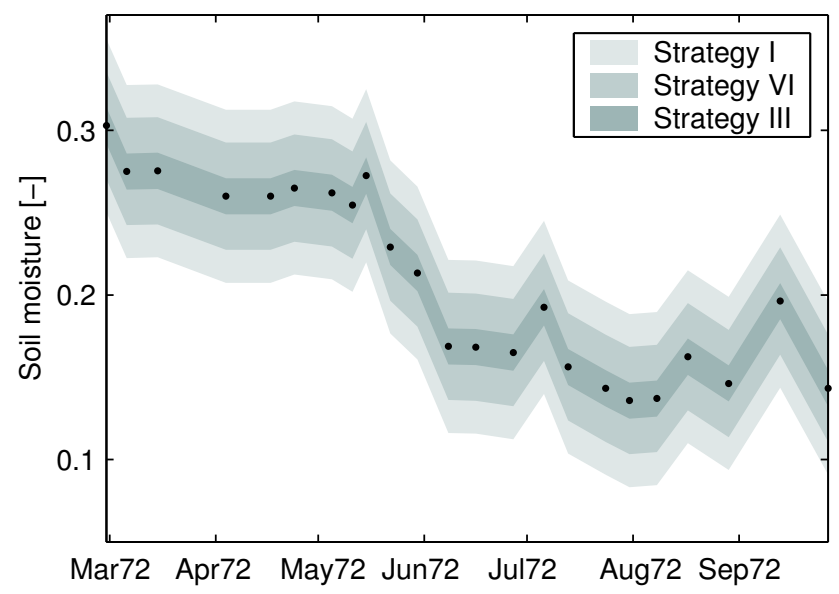

Fig. 10. Approximate $95 \%$ confidence bounds for the spatial mean during a wet-dry transition at R-5, as estimated from soil moisture at a single site (black dots, in this case site 31 ). The outer bounds correspond to the spatial variability, the middle bounds to temporal variability, and the inner bounds give the uncertainty associated with the best linear regression with the spatial mean.

a mean $\mathrm{E}[X \mid i]=\mu_{i}$ and variance $\operatorname{Var}[X \mid i]=\sigma_{i}^{2}$. Here we assume $i$ to be a discrete random variate with uniform probability of $1 / n$. The mean of $X$ is:

$\mathrm{E}[X]=\mathrm{E}[\mathrm{E}(X \mid i)]=\frac{1}{n} \sum_{i=1}^{n} \mathrm{E}[X \mid i]$

The variance of $X$ can be calculated from:

$\operatorname{Var}[X]=\mathrm{E}\left[X^{2}\right]-\mathrm{E}^{2}[X]$

Since

$$
\begin{aligned}
\mathrm{E}\left[X^{2}\right] & =\frac{1}{n} \sum_{i=1}^{n} \mathrm{E}\left[X^{2} \mid i\right] \\
& =\frac{1}{n} \sum_{i=1}^{n}\left\{\operatorname{Var}[X \mid i]+\mathrm{E}^{2}[X \mid i]\right\}
\end{aligned}
$$

the variance of $X$ can also be written as:

$$
\begin{aligned}
\operatorname{Var}[X]= & \frac{1}{n} \sum_{i=1}^{n} \operatorname{Var}[X \mid i]+\frac{1}{n} \sum_{i=1}^{n} \mathrm{E}^{2}[X \mid i] \\
& -\left\{\frac{1}{n} \sum_{i=1}^{n} \mathrm{E}[X \mid i]\right\}^{2}
\end{aligned}
$$

The last term of this equation is expanded as:

$$
\begin{aligned}
\left\{\frac{1}{n} \sum_{i=1}^{n} \mathrm{E}[X \mid i]\right\}^{2}= & \frac{1}{n^{2}} \sum_{i=1}^{n} \mathrm{E}^{2}[X \mid i] \\
& +\frac{2}{n^{2}} \sum_{i=1}^{n} \sum_{j=1}^{i-1} \mathrm{E}[X \mid i] \mathrm{E}[X \mid j]
\end{aligned}
$$


so that combining ( $\mathrm{x})$ and $(\mathrm{x})$ yields an expression that allows $\operatorname{Var}[X]$ to be calculated from the individual $\mathrm{E}[X \mid i]$ and $\operatorname{Var}[X \mid i]$ :

$\operatorname{Var}[X]=\frac{1}{n} \sum_{i=1}^{n} \sigma_{i}^{2}+\frac{n-1}{n^{2}} \sum_{i=1}^{n} \mu_{i}^{2}-\frac{2}{n^{2}} \sum_{i=1}^{n} \sum_{j=1}^{i-1} \mu_{i} \mu_{j}(\mathrm{~A} 6)$

Finally, if $\mathrm{E}[X \mid i]=\mathrm{E}[X \mid j] \quad \forall j \neq i$, then simply $\operatorname{Var}[X]=\frac{1}{n} \sum_{i=1}^{n} \sigma_{i}^{2}$.

Acknowledgements. We thank $\mathrm{K}$. Loague for sharing his data with us. This research is supported by the Wageningen Institute for Environment and Climate Research (WIMEK) and the project Development of a European Land Data Assimilation System to predict Floods and Droughts (ELDAS, EVG1-CT-2001-00050). R. Uijlenhoet acknowledges financial support from the Netherlands Organization for Scientific Research (NWO) through a Innovational Research Incentives Scheme grant (Project 016.021.003).

Edited by: J. C. Refsgaard

\section{References}

Al-Kaisi, M., Brun, L., and Enz, J.: Transpiration and evapotranspiration from maize as related to leaf area index, Agric. Forest Meteorol., 48, 111-116, 1989.

Albertson, J. and Montaldo, N.: Temporal dynamics of soil moisture variability: 1. Theoretical basis, Water Resour. Res., 39, 1274, doi:10.1029/2002WR001616, 2003.

Anderson, M. and Kneale, P.: Topography and hillslope soil water relationhips in a catchment of low relief, J. Hydrol., 47, 115-128, 1980.

Baldocchi, D., Falge, E., Gu, L., Olson, R., Hollinger, D., Running, S., Anthoni, P., Bernhofer, C., Davis, K., Evans, R., Fuentes, J., Goldstein, A., Katul, G., Law, B., Lee, X., Malhi, Y., Meyers, T., Munger, W., Oechel, W., Paw, K., Pilegaard, K., Schmid, H., Valentini, R., Verma, S., Vesala, T., Wilson, K., and Wofsy, S.: FLUXNET: A new tool to study the temporal and spatial variability of ecosystem-scale carbon dioxide, water vapor, and energy flux densities, Bull. Am. Meteorol. Soc., 82, 2415-2434, 2001.

Bárdossy, A. and Lehmann, W.: Spatial distribution of soil moisture in a small catchment. Part 1: geostatistical analysis, J. Hydrol., 206, 1-15, 1998.

Bell, K., Blanchard, B., Schmugge, T., and Witczak, M.: Analysis of surface moisture variations within large-field sites, Water Resour. Res., 16, 796-810, 1980.

Chen, Y.: Letter to the Editor on "rank stability or temporal stability”, Soil Sci. Soc. Am. J., 70, 306, doi:10.2136/sssaj2005.02901, 2006.

Comegna, V. and Basile, A.: Temporal stability of spatial patterns of soil water storage in a cultivated Vesuvian soil, Geoderma, 62, 299-310, 1994.

Cosh, M., Stedinger, J., and Brutsaert, W.: Variability of surface soil moisture at the watershed scale, Water Resour. Res., 40, W12513, doi:10.1029/2004WR003487, 2004.

Crave, A. and Gascuel-Odoux, C.: The influence of topography on time and space distribution of soil surface water content, Hydrol. Process., 11, 203-210, 1997.
Famiglietti, J., Rudnicki, J., and Rodell, M.: Variability in surface moisture content along a hillslope transect: Rattlesnake Hill, Texas, J. Hydrol., 210, 259-281, 1998.

Fitzjohn, C., Ternan, J., and Williams, A.: Soil moisture variability in a semi-arid gully catchment: implications for runoff and erosion control, Catena, 32, 55-70, 1998.

Gómez-Plaza, A., Alvarez-Rogel, J., Albaladejo, J., and Castillo, V.: Spatial patterns and temporal stability of soil moisture across a range of scales in a semi-arid environment, Hydrol. Processes, 14, 1261-1277, 2000.

Grant, L., Seyfried, M., and McNamara, J.: Spatial variation and temporal stability of soil water in a snow-dominated, mountain catchment, Hydrol. Processes, 18, 3493-3511, doi:10.1002/hyp. 5798, 2004.

Grayson, R. and Western, A.: Towards areal estimation of soil water content from point measurements: time and space stability of mean response, J. Hydrol., 207, 68-82, 1998.

Grayson, R., Western, A., Chiew, F., and Blöschl, G.: Preferred states in spatial soil moisture patterns: Local and nonlocal controls, Water Resour. Res., 33, 2897-2908, 1997.

Hawley, M., Jackson, T., and McCuen, R.: Surface soil moisture variation on small agricultural watersheds, J. Hydrol., 62, 179_ 200, 1983.

Hupet, F. and Vanclooster, M.: Intraseasonal dynamics of soil moisture variability within a small agricultural maize cropped field, J. Hydrol., 261, 86-101, 2002.

Hupet, F. and Vanclooster, M.: Sampling strategies to estimate field areal evapotranspiration fluxes with a soil water balance approach, J. Hydrol., 292, 262-280, doi:10.1016/j.jhydrol.2004.01. 006, 2004.

Hupet, F. and Vanclooster, M.: Micro-variability of hydrological processes at the maize row scale: implications for soil water content measurements and evapotranspiration estimates, J. Hydrol., 303, 247-270, doi:10.1016/j.jhydrol.2004.07.017, 2005.

Hupet, F., Bogaert, P., and Vanclooster, M.: Quantifying the localscale uncertainty of estimated actual evapotranspiration, Hydrol Processes, 18, 3415-3434, doi:10.1002/hyp.1504, 2004.

Jacobs, J., Mohanty, B., Hsu, E., and Miller, D.: SMEX02: Field scale variability, time stability and similarity of soil moisture, Remote Sens. Environ., 92, 436-446, doi:10.1016/j.rse.2004.02. 017, 2004.

Kachanoski, R. and de Jong, E.: Scale dependence and the temporal persistence of spatial patterns of soil water storage, Water Resour. Res., 24, 85-91, 1988.

Loague, K.: Soil water content at R-5. Part 1. Spatial and temporal variability, J. Hydrol., 139, 233-251, 1992.

Martínez-Fernández, J. and Ceballos, A.: Mean soil moisture estimation using temporal stability analysis, J. Hydrol., 312, 28-38, doi:10.1016/j.jhydrol.2005.02.007, 2005.

Mohanty, B. and Skaggs, T.: Spatio-temporal evolution and timestable characteristics of soil moisture within remote sensing footprints with varying soil, slope, and vegetation, Adv. Water Resour., 24, 1051-1067, 2001.

Nyberg, L.: Spatial variability of soil water content in the covered catchment at Gårdsjön, Sweden, Hydrol. Processes, 10, 89-103, 1996.

Pachepsky, Y., Guber, A., and Jacques, D.: Temporal persistence in vertical distributions of soil moisture contents, Soil Sci. Soc. Am. J., 69, 347-352, 2005. 
Petrone, R., Price, J., Carey, S., and Waddington, J.: Statistical characterization of the spatial variability of soil moisture in a cutover peatland, Hydrol. Processes, 18, 41-52, doi:10.1002/hyp.1309, 2004.

Price, A. and Bauer, B.: Small-scale heterogeneity and soilmoisture variability in the unsaturated zone, J. Hydrol., 70, 277293, 1984.

Qiu, Y., Fu, B., Wang, J., and Chen, L.: Spatial variability of soil moisture content and its relation to environmental indices in a semi-arid gully catchment of the Loess Plateau, China, J. Arid Environ., 49, 723-750, 2001.

Reynolds, S.: The gravimetric method of soil moisture determination, Part III, An examination of factors influencing soil moisture variability, J. Hydrol., 11, 288-300, 1970.

Ryu, D. and Famiglietti, J.: Characterization of footprint-scale surface soil moisture variability using Gaussian and beta distribution functions during the Southern Great Plains 1997 SGP97 hydrology experiment, Water Resour. Res., 41, W12433, doi: 10.1029/2004WR003835, 2005.

Schenk, H. and Jackson, R.: The global biogeography of roots, Ecol. Monogr., 72, 311-328, 2002.

Schume, H., Jost, G., and Katzensteiner, K.: Spatial-temporal analysis of the soil water content in a mixed Norway spruce (Picea abies (L.) Karst.) - European beech (Fagus sylvatica L.) stand, Geoderma, 112, 273-287, 2003.

Seyfried, M.: Spatial variability constraints to modeling soil water at different scales, Geoderma, 85, 231-254, 1998.
Svetlitchnyi, A., Plotnitskiy, S., and Stepovaya, O.: Spatial distribution of soil moisture content within catchments and its modelling on the basis of topographic data, J. Hydrol., 277, 50-60, doi:10.1016/S0022-1694(03)00083-0, 2003.

Teuling, A. and Troch, P.: Improved understanding of soil moisture variability dynamics, Geophys. Res. Lett., 32, L05404, doi:10. 1029/2004GL021935, 2005.

Vachaud, G., Passerat De Silans, A., Balabanis, P., and Vauclin, M.: Temporal stability of spatially measured soil water probability density function, Soil Sci. Soc. Am. J., 49, 822-828, 1985.

Western, A. and Grayson, R.: The Tarrawarra data set: soil moisture patterns, soil characteristics, and hydrological flux measurements, Water Resour. Res., 34, 2765-2768, 1998.

Western, A., Blöschl, G., and Grayson, R.: Geostatistical characterisation of soil moisture patterns in the Tarrawarra catchment, J. Hydrol., 205, 20-37, 1998.

Western, A., Grayson, R., Blöschl, G., Willgoose, G., and McMahon, T.: Observed spatial organization of soil moisture and its relation to terrain indices, Water Resour. Res., 35, 797-810, 1999.

Wilson, D., Western, A., and Grayson, R.: Identifying and quantifying sources of variability in temporal and spatial soil moisture observations, Water Resour. Res., 40, W02507, doi:10.1029/2003WR002306, 2004.

Wilson, D., Western, A., and Grayson, R.: A terrain and data-based method for generating the spatial distribution of soil moisture, Adv. Water Resour., 28, 43-54, doi:10.1016/j.advwatres.2004. 09.007, 2005.

Wood, E.: Effects of soil moisture aggregation on surface evaporative fluxes, J. Hydrol., 190, 397-412, 1997. 